\title{
An HIV Prevention and Treatment Strategy for Serodiscordant Black Men who have Sex with Men Couples: Using Pre-Exposure Prophylaxis (PrEP) and Antiretroviral Treatment (ART) to Reduce Infections in the Present and the Future \\ Sheldon Applewhite*
}

Department of Sociology, City University of New York, Borough of Manhattan Community College, New York, USA

Last week at the $16^{\text {th }}$ International AIDS Conference in Durban, South Africa, findings were presented from the PARTNERS Study on the use of PrEP and ART among serodiscordant heterosexual couples. The PARTNERS Study is an open-label, prospective interventional study of integrated ART and PrEP delivery for HIV prevention among heterosexual HIV serodiscordant couples. The project was conducted across four sites in Africa, Kisumu and Thika in Kenya and Kabwohe and Kampala in Uganda. The goal was to evaluate, using implementation science methods, a scalable delivery system for PrEP and ART for HIV prevention in couples using counseling, an couple follow-up that could be implemented in a public health clinical setting. The study took place initially in November 2012 and was followed up in June 2016 by the team researchers [1]. The study has important implications towards a better understanding of HIV prevention strategies for couples in the United States among two important groups: serodiscordant couples and African Americans men who have sex with men in primary relationships. The findings from this study should shape the prevention efforts in the United States and could contribute to a turning of the tides of HIV risk and infections in the United States, especially among Black men who have sex with men (MSM) within and outside of primary relationships by addressing the link between the infection rates that arise while in serodiscordant or serostatus unknown relationship. One strategy that has been largely ignored is a couples-based HIV prevention and treatment strategy that is culturally tailored to Black men who have sex with men in primary relationships. Prevention among Black serodiscordant MSM couples also has important implications towards reducing the heavy HIV burden that disproportionately affects African American MSMs.

By increasing efforts to test, counsel and treat Black gay men in relationships with a partner who may be positive or do not know their HIV status, and who may unintentionally infect their current and future partners, we can potentially reduce the HIV infection rates among Black MSMs overall by reducing the levels of HIV within their sexual network through PrEP and ART treatments for those who are negative and positive alike. By increasing the uptake of HIV testing among Black MSM couples whose HIV status is serodiscordant or sero-unkown, it allows for more individuals to receive ART if HIV positive and become engaged in care, and prevention through the use of PrEP for HIV negative members of couples. After the dissolution of the relationship should this occur, HIV infection can be prevented from happening unintentionally once the relationship dissolves and each partner is on PrEP on ART as they move on to their next sexual relationship in the future. This paper addresses the importance of designing a culturally-tailored intervention for Black MSM couples that seeks to identify their HIV status and provide PrEP or ART treatment depending on the status of the partners. The paper also discusses the potential implications for HIV prevention among Black MSMs in the United States.

\section{Background}

Recent studies show that ART and PrEP use substantially reduce HIV risk, by more than $90 \%$ when used with high adherence $[1,2]$. In 2014, the World Health Organizations recommended PrEP and ART as an effective prevention tool for HIV serodiscordant couples. According to the Center for Disease and Prevention, developing effective strategies to deliver ART and PrEP to couples is a key priority in the United States to reduce HIV infections and using ART and PrEP has the potential to significantly increase infection rates in the U.S. However, neither treatment strategy is a panacea and should be used together to maximize the reduction of new HIV infections. For example, several studies on the delivery of ART argue that delays in its use by HIV positive persons is often delayed because they do not know their HIV status or do not immediately seek treatment once testing positive and may engage in HIV risk behaviors long after beginning ART. In terms of PrEP, previous research shows that many at risk individuals will; use PrEP when offered it, but just as many at-risk persons may not [1].

Black gay men as a group have been particularly hard hit by HIV/ AIDS, with HIV prevalence and incidence rates being significantly higher than any other racial or ethnic group of MSM in the United States [3]. However, Black gay men are less likely to know their HIV status, do not test routinely, receive treatment later after receiving a HIV positive diagnosis and are more likely to die from HIV related complications. Moreover, Black gay men, like other gay men, may unknowingly infect their sexual partner(s) if they are unaware of their HIV status.

Although $40 \%$ of individuals engaged in HIV testing and counseling in 2011, couples-based HIV testing, counseling and treatment is low [4,5]. Among serodiscordant couples, HIV testing, counseling and treatment is difficult, with estimates of $20 \%$ to $50 \%$ worldwide of people living with HIV/AIDS unaware of their partners' serostatus [4]. Evidence shows that increasing, voluntary HIV testing, counseling, and treatment among discordant couples significantly increases one's knowledge of partners HIV status, establishes a linkage to care for newly diagnosed HIV-positive persons, create opportunities

*Corresponding author: Sheldon Applewhite, Ph.D., Assistant Professor of Sociology, City University of New York, Borough of Manhattan Community College, 199 Chambers Street, New York, NY 10007, USA, Tel: 212-220-1257; Fax: 212 748-7731; E-mail: sapplewhite@bmcc.cuny.edu

Received July 30, 2016; Accepted August 04, 2016; Published August 11, 2016

Citation: Applewhite S (2016) An HIV Prevention and Treatment Strategy for Serodiscordant Black Men who have Sex with Men Couples: Using Pre-Exposure Prophylaxis (PrEP) and Antiretroviral Treatment (ART) to Reduce Infections in the Present and the Future. J AIDS Clin Res 7: 609. doi: 10.4172/2155-6113.1000609

Copyright: (c) 2016 Applewhite S. This is an open-access article distributed under the terms of the Creative Commons Attribution License, which permits unrestricted use, distribution, and reproduction in any medium, provided the original author and source are credited. 
Citation: Applewhite S (2016) An HIV Prevention and Treatment Strategy for Serodiscordant Black Men who have Sex with Men Couples: Using Pre-Exposure Prophylaxis (PrEP) and Antiretroviral Treatment (ART) to Reduce Infections in the Present and the Future. J AIDS Clin Res 7: 609. doi: 10.4172/2155-6113.1000609

Page 2 of 2

for serodiscordant romantic couples to discuss their HIV statuses and begin or continue discourse related to the one another's and the health of the relationship, and allows couples to make informed, consensual decisions regarding sexual behavior and practices [4,6]. Further, studies show that couples who receive HIV testing, counseling and treatment together if needed are significantly more likely to reduce HIV risk behaviors than those who test alone [4].

Research studies on interventions for serodiscordant couples reveal that they are efficacious in regards to condom use in heterosexual couples [1,7]. Additional studies have shown that providing HIV testing, counseling and treatment to serodiscordant couples is a strategy used to prevent HIV transmission to the negative partner and to ensure that the positive partner is engaged in care. Further, HIV testing counseling, and treatment is an important strategy that links all couples to appropriate prevention, care and treatment services based on the couples' HIV status $[3,8]$. However, research indicates that gay couples seek HIV testing, counseling and treatment far less than their single counterparts, in part, because they perceive themselves at less risk for HIV than single gay men [5,9]. Research shows that gay couples may engage in riskier behavior than gay men [10].

Further, empirical research on serodiscordant couples has been largely limited to focusing on heterosexual couples [1,11] and couples in developing countries [7].

Few specific strategies tailored to the socio-cultural and relationship experiences of Black gay couples have been conducted. There is a dearth in the research on Black MSM couples, testing and PrEP and ART usage. For instance, one report showed that $68 \%$ of new HIV infections occur among main partners [12]. CDC surveillance data shows that $60 \%$ of Black gay men test for HIV infection annually [3]. However, little is known about the HIV testing, counseling, and treatment patterns of Black gay couples, and the structural, socio-cultural and relationship factors that influence their attitudes and practices.

Applewhite discusses the impact that socio-cultural factors such as poverty, homelessness, social support and homophobia have on Black gay romantic relationships and their decision to test for HIV together [13]. In addition, Wheeler et al. reported that Black gay men who are motivated and have community support will use and adhere to PrEP at rates over $90 \%$ [14]. The two studies taken together, along with the PARTNERS Study suggest that Black gay male couples who may or may not know their HIV status and who have social support from each other as well as from their community may be more inclined to test for HIV together, receive counseling and receive ART or PrEP treatment if needed [13,14].

\section{Recommendations and Next Steps}

Additional research studies should be conducted that examine HIV prevention efforts for gay couples overall, which look at testing practices including couples-based testing, that is, testing together as an strategy to reduce HIV infections and an opportunity for couples to ensure each other's HIV status, receive counseling together regarding their health and the relationship, and to seek further treatment if needed or reengage in care. Couples-based testing is a part of the Presidents National Strategy on HIV and AIDS priorities, which is a significant start; however more research and funding efforts should be provided to understand couples in the contest of HIV, testing, counseling and treatment especially as it relates to PrEP and ART use. More data is needed and additional efforts to design interventions specifically for gay couples. Moreover, to date, there are no such studies designed for Black gay couples. Prevention experts are missing a key opportunity to learn more about gay couples overall and about a group who are disproportionately impacted by HIV, yet remain committed to having sexual and romantic relationships with one another. Further, unlike other groups of gay couples, Black gay couples face a myriad of sociocultural factors (i.e., spirituality) that may be unique to them within the context of HIV risk and this is coupled with syndemic factors such as poverty, racism, homophobia, and low SES that may heavily impact their relationships and their potential exposure to HIV infection [13]. Overall, more research attention should be paid to Black gay men overall and their lived experiences, but especially relating to HIV, as this group bears the brunt of the HIV burden in the United States. Until this time, the alarming rates of new HIV infections in the Black gay community should be no surprise with little or no research.

\section{References}

1. Baeten JM, Donnell D, Ndase P, Mugo NR, Campbell JD, et al. (2012) Antiretroviral prophylaxis for HIV prevention in heterosexual men and women. N Engl J Med 367: 399-410.

2. Cohen MS, Chen YQ, McCauley M, Gamble T, Hosseinipour MC, et al. (2011) Prevention of HIV-1 infection with early antiretroviral therapy. N Engl J Med 365: 493-505.

3. Millett GA, Crowley JS, Koh H, Valdiserri RO, Frieden T, et al. (2010) A way forward: The National HIVIAIDS Strategy and reducing HIV incidence in the United States. J Acquir Immune Defic Syndr 55 Suppl 2: S144-147.

4. Chakravarty D, Hoff CC, Neilands TB, Darbes LA (2012) Rates of testing for HIV in the presence of serodiscordant UAI among HIV-negative gay men in committed relationships. AIDS Behav 16: 1944-1948.

5. Purcell DW, Mizuno Y, Smith DK, Grabbe K, Courtenay-Quick C, et al. (2014) Incorporating couples-based approaches into HIV prevention for gay and bisexual men: opportunities and challenges. Arch Sex Behav 43: 35-46.

6. Darbes LA, Chakravarty D, Neilands TB, Beougher SC, Hoff CC (2014) Sexual risk for HIV among gay male couples: a longitudinal study of the impact of relationship dynamics. Arch Sex Behav 43: 47-60.

7. El-Bassel N, Gilbert L, Witte S, Wu E, Hunt T, et al. (2010) Couple-based HIV prevention in the United States: advantages, gaps, and future directions. JAIDS Journal of Acquired Immune Deficiency Syndromes 55: S98-S101.

8. Mustanski B, Parsons JT (2014) Introduction to the special section on sexual health in gay and bisexual male couples. Archives of sexual behavior 43: 17.

9. Greene GJ, Andrews R, Kuper L, Mustanski B (2014) Intimacy, monogamy and condom problems drive unprotected sex among young men in serious relationships with other men: A mixed methods dyadic study. Archives of sexual behavior 43:73-87.

10. Davidovich U, de Wit JB, Stroebe W (2004) Behavioral and cognitive barriers to safer sex between men in steady relationships: Implications for prevention strategies. AIDS Educ Prev 16: 304-314.

11. Wu E, El-Bassel N, Donald McVinney L, Fontaine YM, Hess L (2010) Adaptation of a couple-based HIV intervention for methamphetamine-involved African American men who have sex with men. Open AIDS J 4: 123-131.

12. Bouhnik AD, Preau M, Schiltz MA, Lert F, Obadia Y, et al. (2007) Unprotected sex in regular partnerships among homosexual men living with HIV: A comparison between sero-nonconcordant and seroconcordant couples (ANRSEN12-VESPA Study). Aids 21: S43-S48.

13. Applewhite S, Littlefield MB (2016) The role of resilience and anti-resilience behaviors in the romantic lives of black same gender loving (SGI) men. Journal of Black Sexuality and Relationships, 2: 1-38.

14. Wheeler DP, SD Fields, D Borasky (2014) HPTN 073 pre-exposure prophylaxis (PrEP) initiation and adherence among black men who have sex with men (BMSM) in three US cities. DAIDS 1. 